\title{
Hypokalemic Periodic Paralysis: A Case Report
}

\author{
Blessy Rachal Boban, Cillamol K. J, Elena Cheruvil, Sheffin Thomas, Tony Abraham \\ PHARM.D (Doctor of Pharmacy) Interns, Pushpagiri Medical College Hospital, Thiruvalla, Kerala, India
}

\begin{abstract}
How to cite this paper: Blessy Rachal Boban | Cillamol K. J | Elena Cheruvil | Sheffin Thomas | Tony Abraham "Hypokalemic Periodic Paralysis: A Case Report" Published in International Journal of Trend in Scientific Research and Development (ijtsrd), ISSN: 24566470, Volume-3 । Issue-3, April 2019, pp.216-217, URL: http://www.ijtsrd.co $\mathrm{m} /$ papers/ijtsrd216 58.pdf

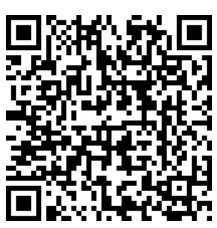

IITSRD21658
\end{abstract}

Copyright (C) 2019 by author(s) and International Journal of Trend in Scientific Research and Development Journal. This is an Open Access article distributed under the terms of the Creative Commons

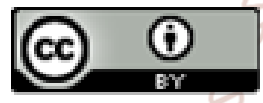
Attribution License (CC BY 4.0) (http://creativecommons.org/licenses/ by/4.0)

\section{INTRODUCTION}

Hypokalemic periodic paralysis (HPP), the most common, form of periodic paralysis, is reasonably rare with an approximated prevalence of 1 in 100,000 [1]. HPP is a condition in which affected individuals may experience flaccid paralytic episodes with concomitant hypokalemia. The clinical features of the syndrome vary somewhat depending on the underlying etiology but the most striking feature is the sudden onset of weakness ranging in severity from mild, transient weakness to severe disability resulting in life-threatening respiratory failure. A perturbation of sodium and calcium ion channels results in low potassium levels and muscle dysfunction [2]. Although the serum potassium level is often alarmingly low, other electrolytes are usually normal. Indeed, total body potassium is actually normal with the change in the serum level reflecting a shift of potassium into cells [3]. Electrocardiographic changes are common. Majority of the cases are characterized as familial or primary HPP, whereas sporadic cases are associated with various other conditions such as barium poisoning, hyperthyroidism, renal disorders, endocrinopathies and gastrointestinal potassium losses. Respiration, deglutition and ocular motility are usually affected only in severe attacks. Furthermore, studies have shown HPP does not affect the heart, but can trigger cardiac arrhythmias. Very rarely, patients can develop permanent weakness but this phenomenon is usually observed in young adults [4]. In hypokalemia familial periodic paralysis, the factors that trigger weakness or paralysis are anesthesia, surgery, pregnancy, insulin, alcohol, strenuous exercise, and steroids. [5,6]. The guidelines for care include control of plasma potassium, avoidance of large glucose and salt loads, maintenance of body temperature, acid-base balance, and careful use of neuromuscular blocking agents.[7,8] . Approximately one-third of cases represent new mutations [9]. Treatment consists of diet changes and avoiding things that trigger your attacks. Carbonic anhydrase inhibitors: These medications increase the flow of potassium. Common options include dichlorphenamide (Keveyis) and acetazolamide (Diamox). Potassium supplements: Oral potassium supplements may be given to help stop an attack that's in progress. Your doctor will advise you on proper dosage [10].

\section{CASE REPORT}

A 24 year-old female with significant past medical history presented to the emergency room with sudden onset paralysis. The patient had gone to bed at $11 \mathrm{pm}$ with no weakness and awoke at 3 a.m found unable to move her upper or lower extremities. The weakness was bilateral and involved both the proximal muscles of the shoulders and hips as well as the distal extremities. She had no respiratory or swallowing difficulty and was able to move her neck and facial muscles. She denied any pain or paresthesia. She had her first episode 10 years ago and it was repeated for 21 times till 2019.Prior to this episode, the patient had taken ice cream as a part of colleague's treat. Usually the episodic 
weakness get relieved with I.V infusion but this time, the weakness was prolonged and taken to a local hospital where her potassium correction was made .But repeat potassium was low (1.9) .Hence referred to Pushpagiri Medical College.She did report several episodes of weakness after consuming ice creams. Her mother had been diagnosed with hepatic cellular carcinoma and died 3 years ago.

On examination, the patient's blood pressure was 100/60. No jugular venous distension, goiter or lymphadenopathy were appreciated. Cardiac exam revealed tachycardia with a regular rhythm and no murmurs. Examination of the lungs and abdomen were unremarkable. Neurologic exam revealed Grade 2 power. Routine chemistry, liver enzymes and complete blood count were normal except for a potassium level of $1.9(3.5-5 \mathrm{mmol} / \mathrm{L}), \mathrm{ESR}-52, \mathrm{ALP}-122$. Two hours after initiation of intravenous potassium replacement, the patient's symptoms had completely resolved. Thyroid stimulating hormone (TSH), triiodothyronine (T3) and thyroxine (T4) levels were obtained and revealed a markedly normal value.

\section{DISCUSSION}

There are several types of Periodic Paralysis associated with metabolic and electrolyte abnormalities. Of these, Hypokalemic Periodic Paralysis (HPP) is the most common. Electromyography reveals abnormalities in some patients but is often normal, especially between episodes when no clinically detectable weakness is present. HPP occurs in several settings and the diagnosis may require an extensive search for the underlying etiology since the treatment varies according to the cause. Rarely, HPP can result from substantial gastrointestinal or renal potassium losses. Acetazolamide may prevent the paralytic episodes and antiarrhythmics or beta-blockers may prevent ventricular ectopy, but there is little data available.Most patients do not require treatment but are instructed to avoid paralysis-inducing situations.

\section{CONCLUSION}

This patient presented with sudden onset paralysis and markedly abnormal potassium, ESR. This presentation is typical of Familial Hypokalemic Periodic Paralysis. The paralysis resolved completely following potassium replacement and she began a course of Acetazolamide 250 mg prior to being discharged from the hospital. At the time of discharge, she had no neurologic findings and a normal blood pressure. She has not suffered any further episodes of paralysis .Periodic Paralysis is important to consider when seeing a patient with sudden onset weakness or paralysis, especially those with no history or evidence of other diseases and no significant risk factors for stroke. Failure to properly diagnose and treat Periodic Paralysis can be fatal, but rapid correction of potassium abnormalities can resolve the symptoms quickly and completely. When possible, the underlying cause must be adequately addressed to prevent the persistence or recurrence of paralysis.

\section{REFERENCES}

[1] Fontaine B. Chapter 1 Periodic Paralysis. Advances in Genetics. 63: Academic Press; 2008. p. 3-23.

[2] Jurkat-Rott K, Lerche H, Lehmann-Horn F. Skeletal muscle channelopathies. J Neurol. 2002; 249:14931502. doi: $10.1007 / s 00415-002-0871-5$.

[3] Lin SH, Lin YF, Chen DT, Chu P, Hsu CW, Halperin ML. Laboratory tests to determine the cause of hypokalemia and paralysis. Arch Intern Med. 2004; 164:1561-1566. doi: 10.1001/archinte.164.14.1561.

[4] Fontaine B. Chapter 1 Periodic Paralysis. Advances in Genetics. 63: Academic Press; 2008. p. 3-23

[5] Melnick B, Chang J, Larson CE, Bedger R. Hypokalemic familial periodic paralysis. Anesthesiology. 1983; 58:263-5

[6] 6. Griggs RC, Resnick J, Engel WK. Intravenous treatment of hypokalemic periodic paralysis. Arch Neurol. 1983;40:539-40

[7] Rollman JE, Dickson CM. Anesthetic management of a patient with hypokalemic periodic paralysis for coronary artery bypass surgery. Anesthesiology. $1985 ; 63: 526-527$

[8] 8. Levitt JO. Practical aspects in the management of hypokalemic periodic paralysis. J Transl Med. 2008;6:18.

[9] https://www.uptodate.com/contents/hypokalemicperiodic-paralysis/abstract/10

[10] www.healthline.com/health/hypokalemic-periodicparalysis 